\title{
Model-based patient matching for in-parallel pressure-controlled ventilation
}

\author{
Jin Wai Wong ${ }^{1}$, Yeong Shiong Chiew ${ }^{1 *}$, Thomas Desaive ${ }^{2}$ and J. Geoffrey Chase ${ }^{3}$
}

\section{*Correspondence:}

chiew.yeong.

shiong@monash.edu

${ }^{1}$ School of Engineering,

Monash University Malaysia,

Selangor, Malaysia

Full list of author information

is available at the end of the article

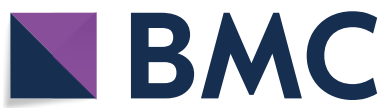

(c) The Author(s) 2022. Open Access This article is licensed under a Creative Commons Attribution 4.0 International License, which permits use, sharing, adaptation, distribution and reproduction in any medium or format, as long as you give appropriate credit to the original author(s) and the source, provide a link to the Creative Commons licence, and indicate if changes were made. The images or other third party material in this article are included in the article's Creative Commons licence, unless indicated otherwise in a credit line to the material. If material is not included in the article's Creative Commons licence and your intended use is not permitted by statutory regulation or exceeds the permitted use, you will need to obtain permission directly from the copyright holder. To view a copy of this licence, visit http:// creativecommons.org/licenses/by/4.0/. The Creative Commons Public Domain Dedication waiver (http://creativecommons.org/publi cdomain/zero/1.0/) applies to the data made available in this article, unless otherwise stated in a credit line to the data. 
sufficient ventilators to support respiratory failure patients [2-4]. Due to the high cost and temporal demand, it is not feasible for a hospital to acquire and maintain this surge capacity of ventilators. Equally, long lengths of MV treatment means hospitals may see shortfalls in ventilators, leading to rationing of care and significant clinical stress, including triaging patients to receive MV care preferentially to others [4].

One solution in shortage of ventilators is to perform in-parallel co-mechanical ventilation (Co-MV) to two or more patients simultaneously. Co-MV, where patients breathe together, has been tested experimentally [5-7] and clinically [8]. To personalise MV settings for each patient, flow restrictors can individualise the flow and volume of air delivered to each patient. The use of high-efficiency particulate air (HEPA) filters can help limit infectious cross-contamination [9]. In addition, installing flow sensors along with additional displays will enable clinicians to monitor and personalise each patient's care [10]. However, Co-MV can offer significant risks due to the inability to individualise MV settings and monitor the personal condition of each patient [11], increasing risk to patients from both over, and under, ventilation [12].

Despite various designs for Co-MV circuits, safety issues still arise whenever clinicians are unsure about the pairing criteria of the patients, and determining the corresponding MV settings for successfully implementing Co-MV. This issue is mainly due to the inability to compensate for variability in patient-specific size and respiratory system mechanics, which vary over the course of disease $[13,14]$. To date, no published studies provide a guideline regarding patient matching and MV settings for Co-MV. Trial-anderror methods are impractical as the repetitive process of switching patients and circuits will increase the risk of other infections and ventilator-induced lung injury (VILI) when patients are mismatched. Thus, it is important to ensure safety if Co-MV is applied.

One proposed Co-MV method is the in-parallel Co-MV. For this Co-MV method, patients must be completely sedated during Co-MV to prevent any spontaneous breathing which would impact on both care and model-based identification of patient-specific respiratory mechanics [15-19]. Similarly, a pressure control (PC) ventilation mode should be used, as it strictly controls peak inspiratory pressure (PIP) and the positive end-expiratory pressure (PEEP) to minimise barotrauma risk, but cannot control peak inspiratory volume [20]. Other key Co-MV parameters include setting respiratory rate (RR) and the ratio of inspiration time to expiration time (I:E).

The major concern of Co-MV in PC mode is the shared tidal volume (VT) delivered to each patient. The distribution of VT depends solely on the patient-specific respiratory mechanics, which vary over time and with patient care [21,22]. Patients with different respiratory mechanics will receive different VT in proportion to these values, and in particular, in proportion to their relative respiratory elastance. A patient with higher respiratory elastance will receive lower tidal volume compared to a patient with lower respiratory system elastance. Thus, during Co-MV, patients with differing elastance may not receive VT within the goal $6-8 \mathrm{~mL} / \mathrm{kg}$ range, increasing the risk of volutrauma [20, 23, 24]. To ensure safe VT distribution, patients must be matched for elastance using a validated respiratory mechanics model able to identify this value and corresponding resistance.

In this research, we present a model-based method to help guide clinical decisionmaking in matching patients for in-parallel Co-MV, at least over short periods of time 
before patient state changes. This method extends existing Co-MV circuit recommendation through the identification of matching patients for Co-MV using patient-specific respiratory mechanics from a well-validated respiratory mechanics model. This method then uses simulation to determine the best set of MV settings to guide Co-MV care. Two outcomes are provided by the proposed model-based approach. First, the matching of suitable pairs of patients to undergo Co-MV. Second, determining the corresponding MV settings for Co-MV with one ventilator and two patients. In addition, a web application with a graphical user interface (GUI) has been developed and available online to accelerate the sharing of the proposed model.

\section{Results}

\section{Pairing patient selection}

The simulated tidal volume obtained from the single-compartment model (SCM) (refer to Methodology Eq. (1)) is presented in resistance-elastance tidal volume contour plots (R-E plot) in Fig. 1a-c, showing the distribution of VT based on different respiratory mechanics using the MV settings shown in Table 3 of the methodology section. Figure 1a shows the R-E contour plot for patient weighing at $50 \mathrm{~kg}$, Fig. $1 \mathrm{~b}$ at $75 \mathrm{~kg}$ and Fig. $1 \mathrm{c}$ at $100 \mathrm{~kg}$.

The green zone is denoted as a safe zone for Co-MV where VT falls in the range of 6-8 $\mathrm{mL} / \mathrm{kg}$. The gradual change from green-to-yellow, yellow-to-orange, and orangeto-red zones represent changes from $\mathrm{VT}=6-8 \mathrm{~mL} / \mathrm{kg}$ to higher tidal volume (moving towards the bottom left of each figure), or to lower tidal volume (moving towards the upper right of each figure). Figure 1 also shows that the region of $6-8 \mathrm{~mL} / \mathrm{kg}$ (centre
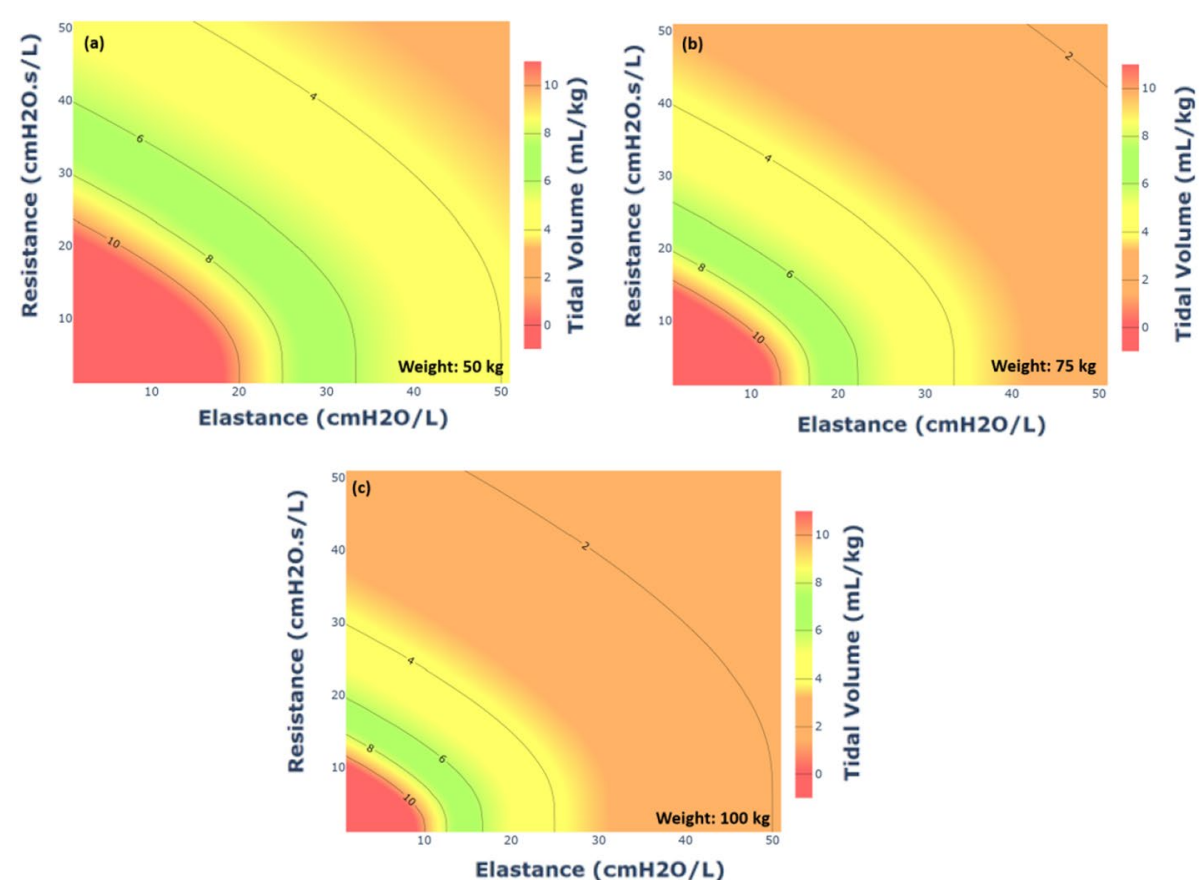

Fig. 1 Resistance-elastance tidal volume contour plots for patient's weight at $50 \mathrm{~kg}(\mathbf{a}), 75 \mathrm{~kg}(\mathbf{b})$, and $100 \mathrm{~kg}$ 
green) reduces if a patient weight increases. For a 100-kg patient in Fig. 1c, the ranges of patient-specific E and R for Co-MV with Patient 1 are highly restricted, as expected due to a narrower range of safe tidal volume. At low resistance values in each figure, the curve is nearly vertical as the change in VT is primarily a function of elastance when $\mathrm{R}$ is low. As $\mathrm{R}$ increases, VT is more sensitive, as this term in Eq. (1) plays a greater role. Thus, the R-E contour plots are not only MV setting-specific, but are also patient weight-specific.

Figure $2 \mathrm{a}-\mathrm{d}$ shows the contour plots for patient weights at $50 \mathrm{~kg}, 65 \mathrm{~kg}, 85 \mathrm{~kg}$ and $100 \mathrm{~kg}$. The patients in Tables 3 and 4 are indicated in their respective weight-specific R-E plots. The VT for each patient in Tables 3 and 4 is presented using their corresponding weight. Based on Fig. 2b, the resultant VT of Patient 1 is $\sim 6.99 \mathrm{~mL} / \mathrm{kg}$, and the VT of Patients B and D fall outside the green region with tidal volume lower than $6 \mathrm{~mL} /$ $\mathrm{kg}$ at $5.74 \mathrm{~mL} / \mathrm{kg}$ (Patient B) and $5.64 \mathrm{~mL} / \mathrm{kg}$ (Patient D). For Patient B with moderate respiratory failure, the lungs are stiffer (with higher E) than Patient 1 , shifting them to the right. Equally, Patient D with significantly higher resistance is shifted upwards from Patient 1. For Patients B and D, higher inspiratory pressure is required to increase the volume of delivered air to the lungs.

Figure 2a shows the MV setting for mild respiratory failure patient is not suitable for Patient A with a weight of $50 \mathrm{~kg}$ as the VT exceeds $8 \mathrm{~mL} / \mathrm{kg}$. Equally, Fig. 2d shows Patient E who weighs $100 \mathrm{~kg}$ falls in the orange zone and should be eliminated from Co-MV candidates. The results of these two patients further highlight the importance

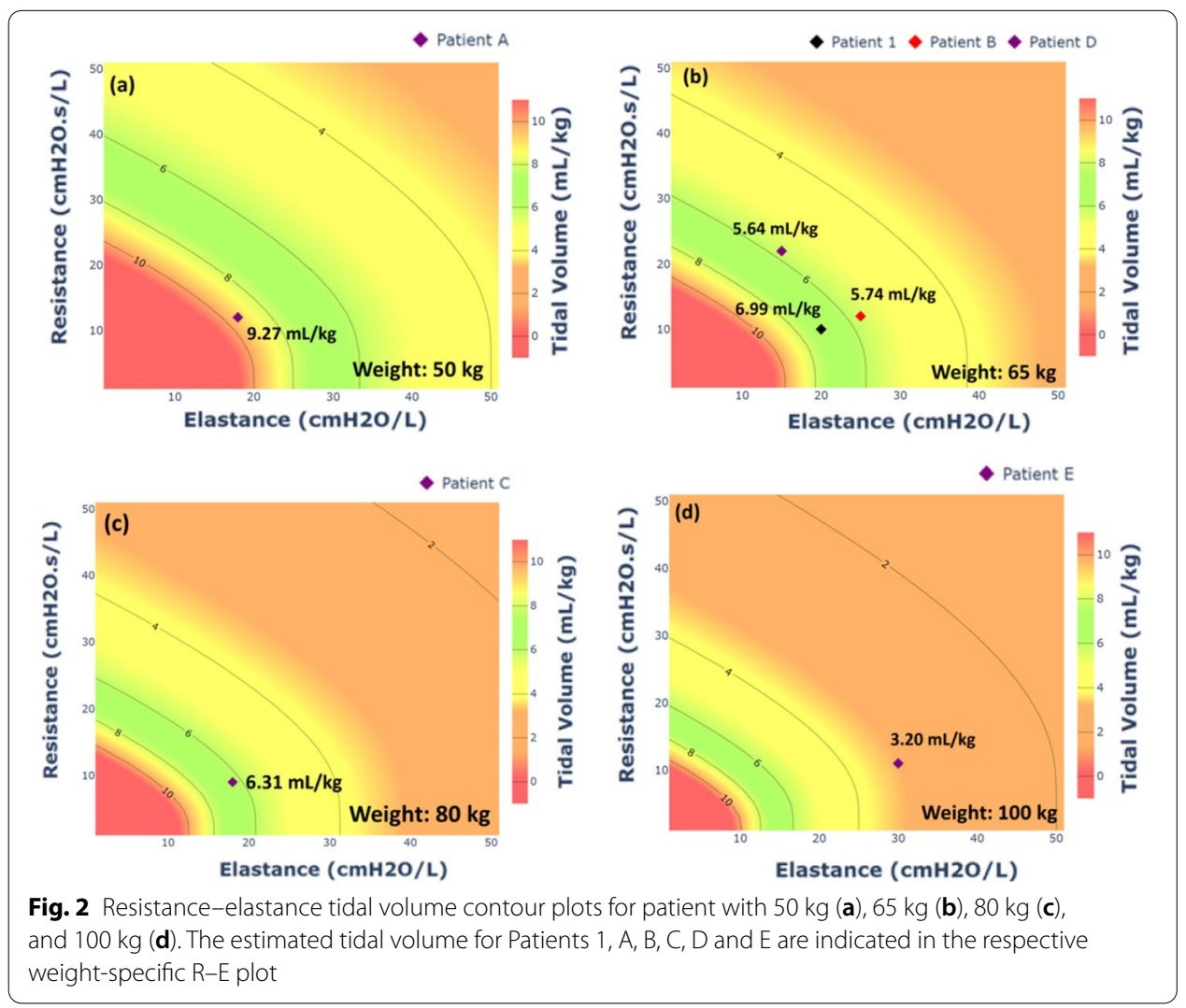


of pairing patients with consideration of predicted body weights. Finally, in this study, the ideal patient to be paired with Patient 1 is Patient $C$, as shown in Fig. 2c with an estimated VT of $\sim 6.31 \mathrm{~mL} / \mathrm{kg}$ when delivered with the same MV settings in Table 3.

\section{Recommended MV settings}

With a decision on pairing Patient 1 with Patient C, the DCM of Eq. (3) is simulated to obtain MV settings for Co-MV. Both Patient 1 and Patient C's respiratory mechanics are used as input to the DCM model to simulate final Co-MV pressure control settings. For the simulation, an arbitrary value of $8 \mathrm{cmH} 2 \mathrm{Os} / \mathrm{L}$ is assigned to $\mathrm{RC}$ of the Co-MV circuit to represent the potential increase of MV circuit resistance due to connection extension to two Patients. The ideal MV pressure settings for Co-MV with the initial inputs (PJ)(t), V1(t), V2(t), V1(t), V2(t)) computed from SCM are shown in Fig. 3.

The ideal PJ(t) (purple line in Fig. 3a) represents the pressure graph generated from MV based on the initial MV setting in Table 3. A positive flow rate indicates air entering the lungs during inspiration, whereas a negative flow rate indicates the exiting airflow during expiration. Due to the existence of Rc, the resultant solution for $\mathrm{P}(\mathrm{t})$ has appeared to be an inverse ramp waveform (green line) as shown in Fig. 3a. Theoretically, this is the ideal airway pressure to be delivered by the MV for both Patients 1 and $\mathrm{C}$ for them to receive the desired VT. Unfortunately, modern pressure-controlled MV only generates square wave-like pressure. It is also implausible to apply a negative airway pressure. Thus, to ensure sufficient VT is delivered, the main factor to be

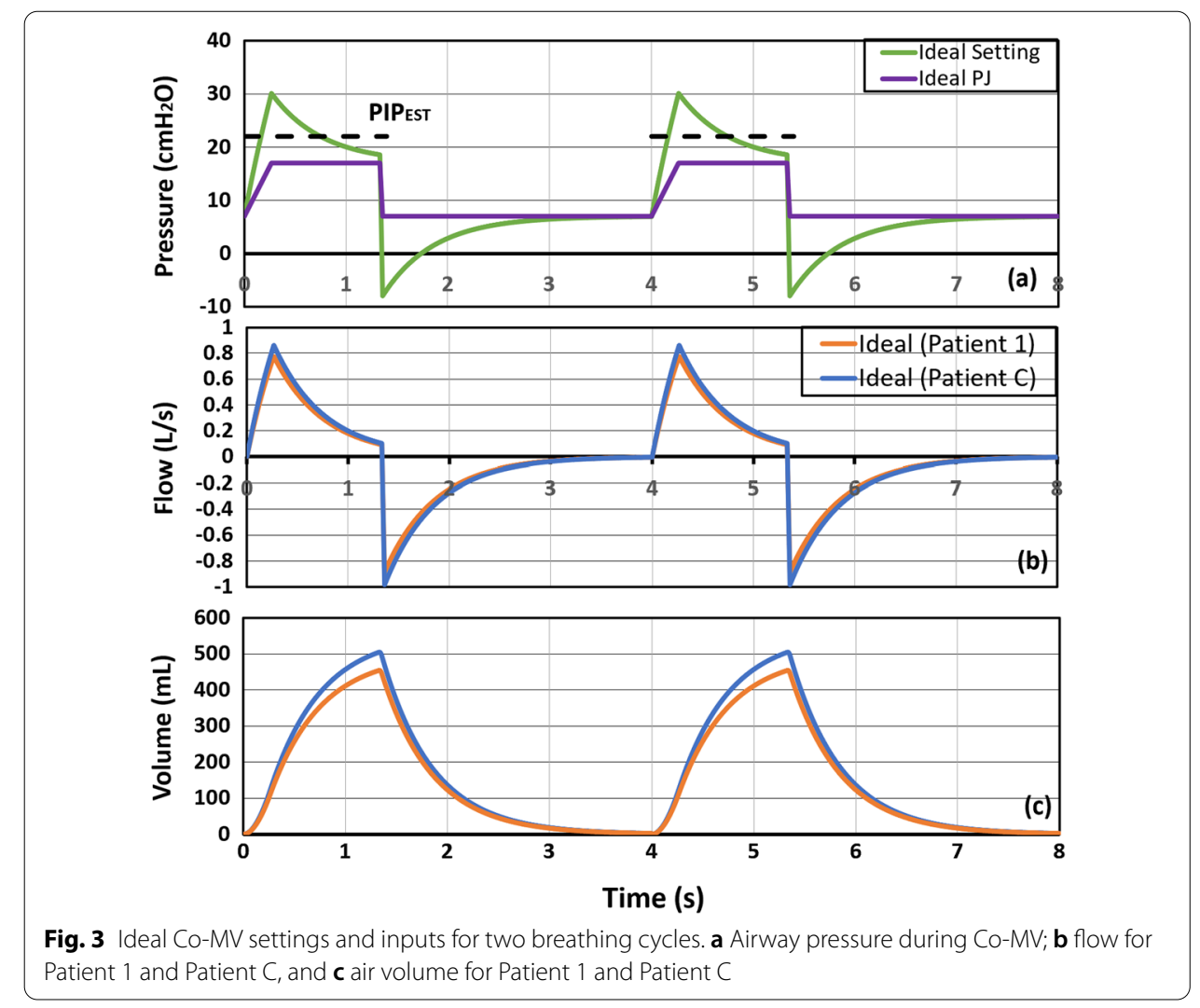


considered is to adjust the inspiratory pressure generated from the ventilator, which can be achieved via simple MV settings.

In this study, the inspiratory pressure is controlled by changing the PIP while keeping the PEEP constant. Based on the ideal $\mathrm{P}(\mathrm{t})$, PIPEST is estimated by taking the average pressure from the ideal setting as shown in Fig. 3a (green solid line), resulting in the PIPEST as the dotted black line. However, this approach will typically lead to a lower VT compared to ideal values. In response, the proposed actual MV setting is obtained through an iterative process. The value of PIPEST obtained from the ideal setting is increased by $0.5 \mathrm{cmH} 2 \mathrm{O}$ for each simulation iteration before a VT with minimum error compared to the desired VT is obtained. Figure 4a (green solid line) shows the finalised actual MV pressure setting for Co-MV after several iterations. Based on the finalised MV setting, PIP has increased to $22.5 \mathrm{cmH} 2 \mathrm{O}$, which is $5.5 \mathrm{cmH} 2 \mathrm{O}$ higher than the initial $\mathrm{MV}$ setting at $17 \mathrm{cmH} 2 \mathrm{O}$. The inspiratory pressure required to deliver the desired VT to each patient is thus equal to $15.5 \mathrm{cmH} 2 \mathrm{O}$ (PIP$\mathrm{PEEP}=15.5 \mathrm{cmH} 2 \mathrm{O})$. As a result, the actual $\mathrm{PJ}(\mathrm{t})$ is no longer a perfect square wave (purple solid line). Compared to the ideal PJ in Fig. 4a, the actual PJ has a higher peak inspiratory pressure.

The peak flow rate in Fig. 4b shows a significant decrease compared to the ideal flow of Fig. 3b. Nonetheless, the subsequent decreasing rate of the actual airflow is lower thus ensuring sufficient air is delivered to the lungs. From Fig. 4c, the ideal VT is $454.2 \mathrm{~mL}$, whereas the actual VT is $458.5 \mathrm{~mL}$, which is a negligible difference at $0.86 \%$ error. For the result of Patient $\mathrm{C}$, the ideal and the actual VT are also very close at $0.95 \%$ error. The summarised results of VT in $\mathrm{mL} / \mathrm{kg}$ are shown in Table 1.

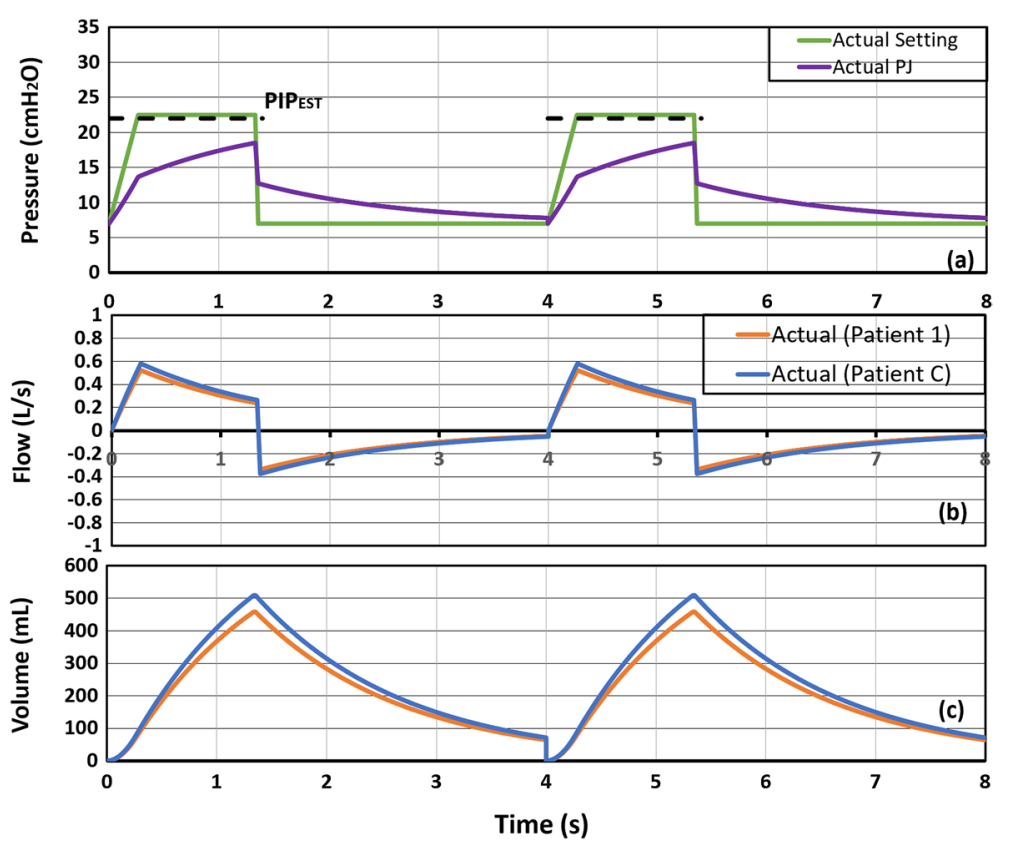

Fig. 4 Proposed actual Co-MV settings for two breathing cycles with the estimated flow and volume: a airway pressure during Co-MV; $\mathbf{b}$ flow for Patient 1 and Patient $\mathrm{C}$, and $\mathbf{c}$ air volume for Patient 1 and Patient $\mathrm{C}$ 
Table 1 Results of VT for patients under Co-MV

\begin{tabular}{lll}
\hline & Patient 1 & Patient C \\
\hline Ideal VT $(\mathrm{mL} / \mathrm{kg})$ & 6.99 & 6.31 \\
Actual VT $(\mathrm{mL} / \mathrm{kg})$ & 7.05 & 6.36 \\
Percentage error $(\%)$ & 0.86 & 0.95
\end{tabular}

\section{Discussion}

\section{General implementation}

The proposed protocol has demonstrated successful patient matching and MV setting in a simulated environment. Apart from finding a suitable patient, further noteworthy information can be obtained from this pairing process. First, due to the highly restrictive respiratory mechanics, obese patients have to be singly ventilated with extra care. This result was not noted in any prior simulation studies, nor in the one clinical case study done [8], and represents a major risk given rising obesity rates, and the greater risk during COVID-19 for patients with this comorbidity.

Aside from providing the estimated VT, the R-E contour plots can provide insights for the clinicians to evaluate the subsequent effects of Co-MV. The R-E tidal volume contour plot enables adjusting patients MV circuit resistance to increase or decrease tidal volume. For example, increasing the airway resistance can shift the VT of Patient A lower so it falls within the green zone. This resistance can be achieved by inserting an adjustable resistor in the inspiratory tube. The added resistor component will thus lower the amount of tidal volume entering Patient A. Such resistors can be placed in the respiratory circuit at the start and left at very low or zero value until needed. Another example is if Patient $\mathrm{C}$ has clinically improved during the process of Co-MV and the value of $\mathrm{E}$ drops to $10 \mathrm{cmH} 2 \mathrm{O} / \mathrm{L}$, their VT is now out of the green zone and might place the patient at risk. With this foreseeable situation, the clinician can be given an alternative to instal an adjustable resistor in the inspiratory path of Patient $\mathrm{C}$ as shown in Fig. 5. This resistor can decrease the potential tidal volume delivered to Patient $\mathrm{C}$.

\section{Future work}

Using the model-based approach, clinicians are able to virtually preview the results of Co-MV by pairing patients in a virtual environment. Similar to the DCM model of Eq. (3), which is also novel to this work, different settings can be tested virtually without causing harm. Nevertheless, this study is conducted within a simulated environment by implementing a purely mathematical model. Experiments must be performed in vitro, followed by animal and/or clinical studies to further validate the efficacy and safety of the proposed model-based method. However, there is no specific physiological reason the system could not work as shown. In this study, the proposed method is compared to a clinical Co-MV study performed by Beitler et al. [8]. The patient-specific information and the ventilator settings from the clinical study are used as input for the proposed method. The resulting tidal volume received by each patient during Co-MV from Beitler et al. is compared to our proposed model simulation. Results show that our simulation yields an average difference of $11.57 \%$ in the VT compared to Beitler et al. study (please 


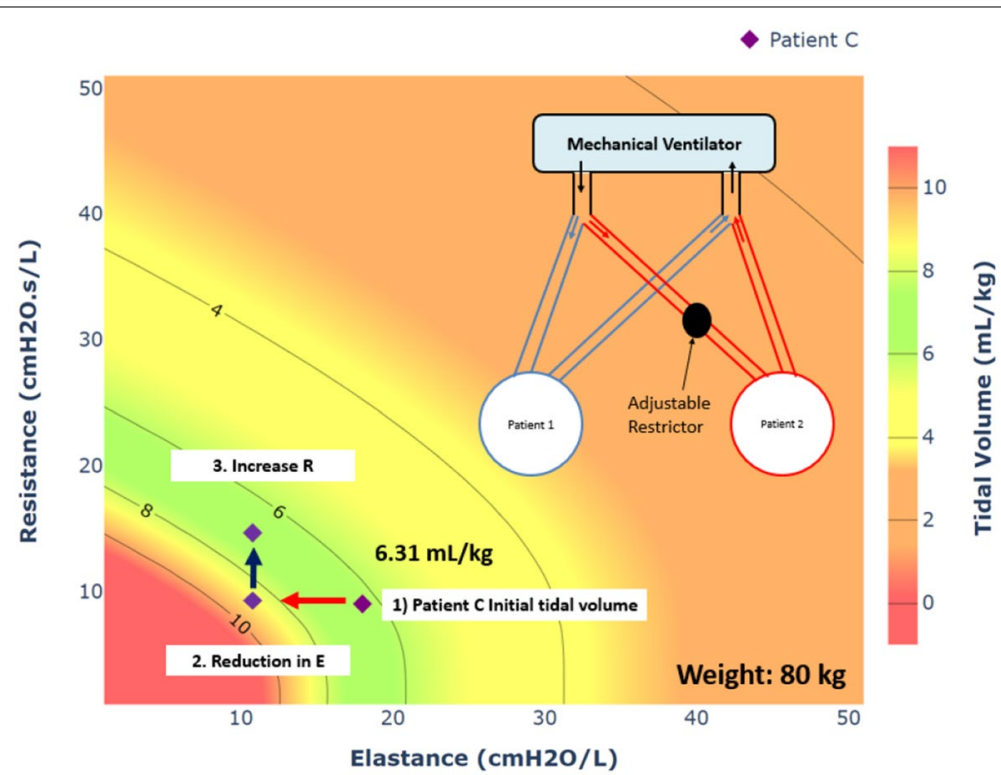

Fig. 5 Resistance-elastance tidal volume contour plots for patient weighing $80 \mathrm{~kg}$. The placement of the adjustable resistor in the parallel Co-MV circuit (Patient C) is shown in the upper right corner. As Patient C's condition improves with lowering of respiratory elastance (E), the tidal volume delivered increases and moved out of the safe green zone. With the increase of resistance (R) in Patient C's Co-MV circuit, the tidal volume range can be shifted back to the green zone

refer to Additional file 1 for the details). We also found these Co-MV patient pairs, if ventilated with a specific ventilator setting, they are within a safe tidal volume range similar to the clinical trial. These results, based on comparison to the one clinical study of patient pairing to date, further highlight the potential of this proposed method in better matching Co-MV patients.

Setting MV for respiratory failure is difficult and it is even cumbersome during Co-MV. Thus, additional MV adjustment factors should be considered in real-life application. For instance, one patient might need a higher fraction of inspired oxygen (FiO2) to increase oxygenation when collapsed alveoli are not recruitable, which is not possible with either in-parallel or in-series Co-MV. The proposed model is unable to provide the adjustment required for the remaining MV parameters other than inspiratory pressure during pressure-controlled Co-MV. Equally, patient-specific parameters are likely to diverge over time as the disease progresses differently for each patient. Such interand intra-patient variability is a major concern in any form of MV $[25,26]$. To overcome the inconsistencies of respiratory system mechanics, the proposed method can be implemented in a closed-loop system capable of monitoring the health conditions of the Co-MV patients in real-time [22, 27-29]. An in-series that enables more control and personalised MV, which would also benefit from patient matching, could be used where each patient has a unique inspiratory circuit and control [30].

Clinically, although the proposed method has demonstrated the ability to assist clinicians during Co-MV, Co-MV should only be treated as a temporary solution and last resort during a health crisis [12]. Thus, any use of this ventilation approach should be restricted or held for short term MV until more capacity can be found. Equally, COVID19 and respiratory failure patients could be avoided for Co-MV, where more stable 
patients requiring MV could be matched, thus reducing some of the potential issues with variability.

\section{Open access}

To accelerate the sharing of the proposed model amid the COVID-19 pandemic, a web application with a graphical user interface (GUI) has been developed by using an opensource programming language, Python. Flask is chosen as the web framework for web app development. In terms of the graphical presentation, the Plotly Python graphical library is implemented. The web application is developed based on the proposed virtual protocol and can be found in the following link (https://multiplex-ventilation.herok uapp.com/pressurecontrol). The web application summary and guide are included in an Additional File 1, along with this manuscript.

\section{Conclusions}

Deciding the patients for co-ventilation is a complex and stressful process. The modelbased approach presented in this study could serve as a guideline to determine a pair of patients and the corresponding MV settings for in-parallel co-mechanical ventilation. By having preliminary results prior to practical application, the risk of causing catastrophic complications and VILI can be decreased significantly. Nevertheless, additional clinical trials are required for further validations.

\section{Methods}

\section{Setting up for co-ventilation (Co-MV)}

In this study, a set of 'safe' PC MV settings is used based on several major clinical studies, as shown in Table 2.

To set up Co-MV, a patient must first be ventilated to obtain the corresponding patient-specific respiratory mechanics, airway resistance (R) and respiratory system elastance (E). These values can provide information on their response to MV and can be identified from measured airway pressure and flow using multiple linear regression $[26,36]$. A clinically validated single-compartment linear lung model (SCM) [26, 3638 ] is then used together with the respiratory mechanics to forward simulate potential mechanical ventilator output. The output can then assist clinicians in selecting the most compatible patients for Co-MV. After finding the suitable patient pair, further simulation using a double-compartment linear lung model (DCM) is conducted to determine the best Co-MV settings for these two specific patients. The summary of performing Co-MV is shown in the flowchart in Fig. 6.

Table 2 Recommended MV settings from literature

\begin{tabular}{lll}
\hline Parameters & Criteria & Clinical study references \\
\hline Plateau pressure, PPLAT & $<35 \mathrm{cmH} 2 \mathrm{O}$ & Gattinoni et al. [31] \\
Positive end-expiratory pressure, PEEP & $5-25 \mathrm{cmH} 2 \mathrm{O}$ & Gattinoni et al. [32] \\
Respiratory rate, RR & $12-20$ breath per min & O'Driscoll et al. [33] \\
Inspiratory expiratory ratio, l:E & $1: 2-1: 5$ & Poor et al. [34] \\
Tidal volume, VT & $6-8 \mathrm{~mL} / \mathrm{kg}$ & ARDSNet [35] \\
\hline
\end{tabular}




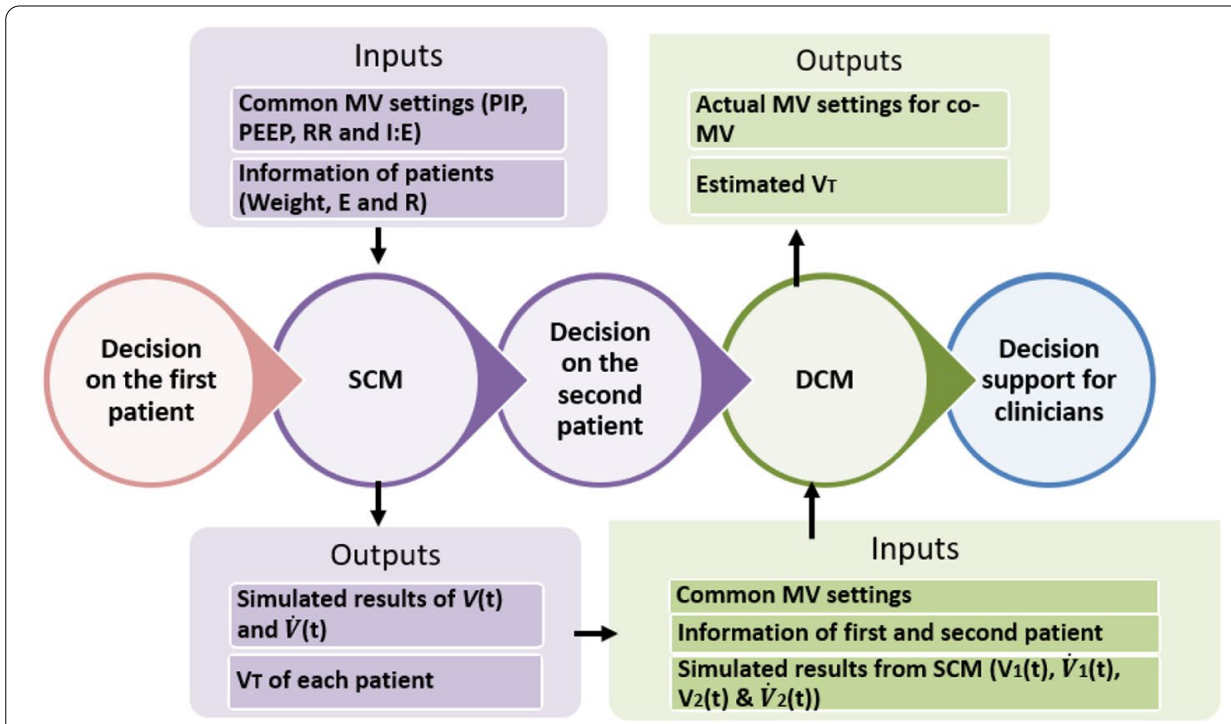

Fig. 6 Flowchart of the protocol stages for Co-MV

\section{Lung compartment models}

A sample setup for in-parallel Co-MV and the equivalent mathematical models to represent the Co-MV setup is shown in Fig. 7. The ventilator circuit (Fig. 7a) can be separated into two circuits in-parallel to connect to two patients. Each patient circuit, Patient 1 (blue) or Patient 2 (red) are fitted with one-way valves to ensure single direction flow. At each expiration circuit, they are fitted with HEPA filters to prevent cross-contamination. Figure 7b shows single-patient ventilation represented by a SCM and Fig. 7c shows the DCM represented an in-parallel Co-MV circuit.

The mathematical model of a single-patient MV circuit can be represented by an electrical circuit as shown in Fig. 7b describing the single-compartment lung model [39]:

$$
\begin{aligned}
& P(t)=E V(t)+R \dot{V}(t)+P_{0}, \\
& \dot{V}(t)+\frac{E V(t)}{R}=\frac{\left(P(t)-P_{0}\right)}{R},
\end{aligned}
$$

where the airway pressure $(\mathrm{P})$ is controlled during $\mathrm{PC}$ ventilation mode, time $(\mathrm{t})$, volume $(\mathrm{V})$, and flow $(\dot{V})$ are measured from the ventilator. The respiratory elastance $(\mathrm{E})$, airway resistance $(\mathrm{R})$ can be determined via multiple linear regression with $\mathrm{P} 0$ assumed as positive end-expiratory pressure (PEEP) when there is no intrinsic PEEP.

For the double-compartment model DCM version, as shown in Fig. 7c, where the second patient shares the input pressure. In the electrical circuit, compliance $(C)$ is the inverse of elastance $(\mathrm{E})$. The parallel circuit for Co-MV with a second patient is thus defined:

$$
\begin{aligned}
& \left(R_{1}+R_{2}\right) \dot{P}(t)+\left(E_{1}+E_{2}\right) P(t) \\
& =\left[R_{1} R_{2}+R_{c}\left(R_{1}+R_{2}\right)\right] \ddot{V}(t) \\
& \quad+\left[\left(R_{c}+R_{2}\right) E_{1}+\left(R_{c}+R_{1}\right) E_{2}\right] \dot{V}(t) \\
& \quad+E_{1} E_{2} V(t),
\end{aligned}
$$




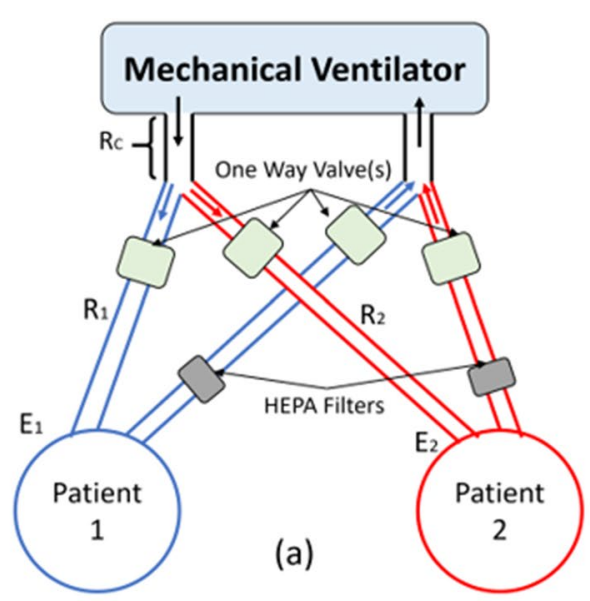

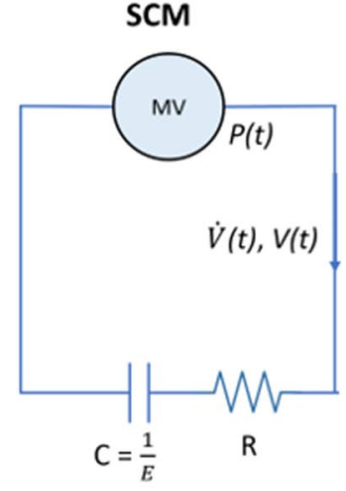

(b)

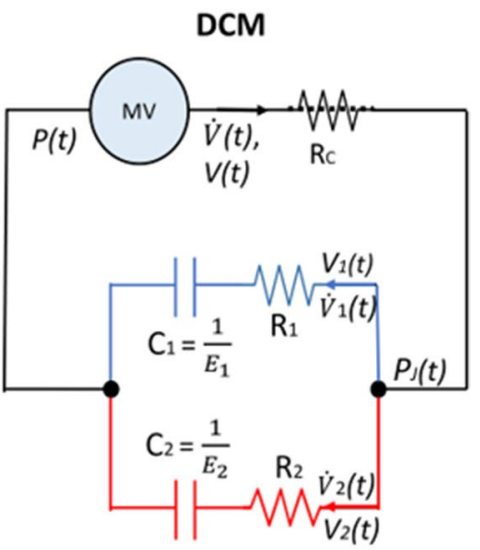

(c)

Fig. 7 Setup for Co-MV (a) and the equivalent mathematical model for single-compartment model (SCM) (b) and double-compartment model (DCM) (c)

Table 3 Patient 1 respiratory mechanics and MV settings

\begin{tabular}{|c|c|c|c|c|}
\hline Patient ID & Respiratory failure & $\mathrm{E}(\mathrm{cmH} 2 \mathrm{O} / \mathrm{L})$ & $\mathrm{R}(\mathrm{cmH} 2 \mathrm{Os} / \mathrm{L})$ & Weight (kg) \\
\hline 1 & Mild & $20(18-27)$ & $10(9-14)$ & 65 \\
\hline \multicolumn{5}{|c|}{ Initial MV settings for Patient 1} \\
\hline \multicolumn{2}{|c|}{ PIP $(\mathrm{cmH} 2 \mathrm{O})$} & & & 17 \\
\hline \multicolumn{2}{|c|}{ PEEP $(\mathrm{cmH} 2 \mathrm{O})$} & & & 7 \\
\hline \multicolumn{2}{|c|}{$\mathrm{RR}(\mathrm{bpm})$} & & & 15 \\
\hline \multicolumn{2}{|l|}{ I:E } & & & $1: 2$ \\
\hline
\end{tabular}

where Patient 1 and Patient 2 are defined in terms of their patient-specific respiratory mechanics (E1, E2, R1, R2) and resulting patient-specific volume and flow (V1(t), V1(t), V2(t), V2(t)) using subscripts, and are seen in the blue and red paths in Fig. 7c. RC is the common resistance component due to the ventilation circuit where it is shared during Co-MV. PJ is the pressure at the joint connecting both patients during Co-MV. 
Table 4 Potential patients to be Co-MV with Patient 1

\begin{tabular}{lllll}
\hline Patient ID & Respiratory failure & $\mathbf{E}(\mathbf{c m H 2 O} / \mathbf{L})$ & $\mathbf{R}(\mathbf{c m H} \mathbf{2 O} \mathbf{s} \mathbf{L})$ & Weight $\mathbf{( k g )}$ \\
\hline A & Normal & $18(15-22)$ & $12(10-15)$ & 50 \\
B & Moderate & $25(20-32)$ & $12(10-14)$ & 65 \\
C & Mild & $18(18-27)$ & $9(9-14)$ & 80 \\
D & Obstructive & $15(13-23)$ & $22(16-33)$ & 65 \\
E & Severe & $30(22-33)$ & $11(9-14)$ & 100 \\
\hline
\end{tabular}

Equation (3) can be solved to obtain the resulting behaviour, where all simulations are performed using MATLAB 2019 (Natick, MA).

\section{Simulation of Co-MV patient pairing}

In this study, we demonstrate the model-based pairing process through simulation of virtual patients [40-43]. A virtual patient, Patient 1 is simulated as the first candidate considered for Co-MV. Table 3 shows Patient 1's respiratory mechanics and the corresponding PC mode MV settings used for their MV care. Five additional virtual patients, Patients A, B, C, D and E with different respiratory mechanics are assigned as potential candidates for Co-MV. These patients' suitability to be paired with Patient 1 are evaluated using model-based predicted tidal volume (in $\mathrm{mL} / \mathrm{kg}$ ) corresponding to patient-specific respiratory mechanics and weights. Solving Eq. (2) with $\mathrm{P}(\mathrm{t}), \mathrm{P} 0, \mathrm{R}$ and $E$, and patient weight as input will enable estimation of each patient's predicted tidal volume.

To ensure the patient-specific values of $E$ and $R$ are realistic, they are chosen based on the values found in the literature [44]. Table 4 shows the respiratory mechanics of the 5 potential virtual patients (A, B, C, D, E) screened for Co-MV with Patient 1. Each patient's respiratory mechanics were set based on different levels of respiratory failure, where a severe respiratory failure results in greater elastance, and obstructive disorders have greater resistance. In this study, the mean values of the patient's respiratory system elastance and resistance are used.

Patient 1 with mild respiratory failure, is the first patient being 'ventilated', with Patients A-E being potential matches. With the common MV setting from Patient 1, and the information of each patient, simulation is conducted with Eq. (2) to select the second patient from among Patients A to E. For a more general solution, values of $\mathrm{E}$ and $\mathrm{R}$ within the range of 1 to 50 will be simulated for the second patient to assess where the best patient match might be. After selecting the second patient, the final step is to simulate the actual MV setting by using the DCM model of Eq. (3). The feasibility of the actual MV setting is evaluated by comparing the percentage difference of VT when co-ventilated.

\footnotetext{
Abbreviations

C: Compliance; Co-MV: Co-mechanical ventilation; DCM: Double compartment lung model; E: Respiratory elastance; FiO2: Fraction of inspired oxygen; GUI: Graphical user interface; HEPA: High efficiency particulate air; I:E: Ratio of inspiration time to expiration time; MV: Mechanical ventilation; P: Airway pressure (P); PO: Offset pressure; PC: Pressure control; PEEP: Positive end-expiratory pressure; PIP: Peak inspiratory pressure; PIPEST: Estimated peak inspiratory pressure; PJ: Pressure at the joint connecting both patients; PPLAT: Plateau pressure; R: Airway resistançe (R); R-E: Resistance-elastance; RR: Respiratory rate; SCM: Single compartment linear lung model; t: Time; V: Volume; $\dot{V}$ : Flow; $\ddot{V}:$ Acceleration; VILI: Ventilator-induced lung injury; VT:Tidal volume.
} 


\section{Supplementary Information}

The online version contains supplementary material available at https://doi.org/10.1186/s12938-022-00983-y.

Additional file 1. Web application.

\section{Acknowledgements}

The authors would like to thank the MedTech Centre of Research Expertise, University of Canterbury, New Zealand, the New Zealand Ministry of Business Innovation and Employment (MBIE) Covid Innovation Action Fund (CIAF), and Monash University Malaysia Advance Engineering Platform (AEP) for supporting this research.

\section{Authors' contributions}

JWW and YSC prepared the manuscript. All authors had input in writing and revising the manuscript. All authors read and approved the final manuscript.

\section{Funding}

Not applicable

Availability of data and materials

Data are available upon request.

\section{Declarations}

Ethics approval and consent to participate

Not applicable.

\section{Consent for publication}

Not applicable.

\section{Competing interests}

The authors declare that they have no competing interests.

\section{Author details}

${ }^{1}$ School of Engineering, Monash University Malaysia, Selangor, Malaysia. ${ }^{2}$ GIGA-In Silico Medicine, University of Liege, Liege, Belgium. ${ }^{3}$ Centre for Bioengineering, University of Canterbury, Christchurch, New Zealand.

Received: 14 October 2021 Accepted: 24 January 2022

Published online: 09 February 2022

\section{References}

1. Penarredonda JL. Covid-19: the race to build coronavirus ventilators. 2020. 2020.

2. Cohen IG, Crespo AM, White DB. Potential legal liability for withdrawing or withholding ventilators during COVID-19: assessing the risks and identifying needed reforms. JAMA. 2020;323(19):1901-2.

3. Ricci M, Gallina P. COVID-19-immunity from prosecution for physicians forced to allocate scarce resources: the Italian perspective. Crit Care. 2020;24(1):295.

4. Truog RD, Mitchell C, Daley GQ. The toughest triage - allocating ventilators in a pandemic. N Engl J Med. 2020;382:1973-5.

5. Neyman G, Irvin CB. A single ventilator for multiple simulated patients to meet disaster surge. Soc Acad Emerg Med. 2006:13:1246-9.

6. Paladino $L$, et al. Increasing ventilator surge capacity in disasters: ventilation of four adult-human-sized sheep on a single ventilator with a modified circuit. Resuscitation. 2008;77(1):121-6.

7. Smith R, Brown J. Simultaneous ventilation of two healthy subjects with a single ventilator. Resuscitation. 2009:80(9):1087.

8. Beitler JR, et al. Ventilator sharing during an acute shortage caused by the COVID-19 Pandemic. Am J Respir Crit Care Med. 2020;202:600-4

9. de Jongh $\mathrm{FH}$, et al. Ventilating two patients with one ventilator: technical setup and laboratory testing. ERJ Open Res. 2020. https://doi.org/10.1183/23120541.00256-2020

10. Srinivasan $\mathrm{S}$, et al. Individualized system for augmenting ventilator efficacy (iSAVE): a rapidly deployable system to expand ventilator capacity. BioRxiv. 2020;13:1246.

11. SCCM, et al. Consensus statement on multiple patients per ventilator. SCCM Website. https://www.sccm.org/Disaster/ Joint-Statement-on-Multiple-Patients-Per-Ventilato; 2020.

12. Chase JG, et al. In-parallel ventilator sharing during an acute shortage: too much risk for a wider uptake. Am J Respir Crit Care Med. 2020;202(9):1316-7.

13. Branson $\mathrm{RD}$, et al. Use of a single ventilator to support 4 patients: laboratory evaluation of a limited concept. Respir Care. 2012;57(3):399-403.

14. Lee JWW, et al., Stochastic modelling of respiratory system elastance for mechanically ventilated respiratory failure patients. Ann Biomed Eng, 2021:1-16.

15. Chatburn RL, Branson RD, Hatipoğlu U. Multiplex ventilation: a simulation-based study of ventilating 2 patients with a single ventilator. Respir Care. 2020;65(7):920-31. 
16. Major $V$, et al. Respiratory mechanics assessment for reverse-triggered breathing cycles using pressure reconstruction. Biomed Signal Process Control. 2016;23:1-9.

17. Chiew YS, et al. Assessing mechanical ventilation asynchrony through iterative airway pressure reconstruction. Comput Methods Programs Biomed. 2018;157:217-24.

18. Gutierrez $\mathrm{G}$, et al. Automatic detection of patient-ventilator asynchrony by spectral analysis of airway flow. Crit Care. 2011;15(4):R167.

19. Kannangara DO, et al. Estimating the true respiratory mechanics during asynchronous pressure controlled ventilation. Biomed Signal Process Control. 2016;30:70-8.

20. Major VJ, et al. Biomedical engineer's guide to the clinical aspects of intensive care mechanical ventilation. Biomed Eng Online. 2018;17(1):169

21. Chiew YS, et al. Feasibility of titrating PEEP to minimum elastance for mechanically ventilated patients. Pilot Feasibility Stud. 2015;1:9.

22. Ng QA, et al. Network data acquisition and monitoring system for intensive care mechanical ventilation treatment. IEEE Access. 2021;9:91859-73.

23. Chao DC, Scheinhorn DJ. Barotrauma vs volutrauma. Chest J. 1996;109(4):1127-8.

24. Ricard JD, Dreyfuss D, Saumon G. Ventilator-induced lung injury. Eur Respir J. 2003;22(42_suppl):2s-9.

25. van Drunen E, et al. Expiratory model-based method to monitor ARDS disease state. Biomed Eng Online. 2013;12(1):57.

26. Morton SE, et al. Optimising mechanical ventilation through model-based methods and automation. Ann Rev Control. 2019;48:369-82

27. Szlavecz A, et al. The Clinical Utilisation of Respiratory Elastance Software (CURE Soft): a bedside software for real-time respiratory mechanics monitoring and mechanical ventilation management. Biomed Eng Online. 2014;13(1):140.

28. Rees, S.E., The Intelligent Ventilator (INVENT) project: The role of mathematical models in translating physiological knowledge into clinical practice. Computer Methods Programs Biomed 2011;104, Supplement 1(0):S1-S29.

29. $\mathrm{Ng}$ QA, et al. Mechanical ventilation monitoring: development of a network data acquisition system. IFAC-PapersOnLine. 2020;53(2):15916-21.

30. Chase JG, et al. Safe doubling of ventilator capacity: a last resort proposal for last resorts. Crit Care. 2020;24(1):222.

31. Gattinoni L, et al. Lung recruitment in patients with the acute respiratory distress syndrome. N Engl I Med. 2006;354(17):1775-86.

32. Gattinoni L, et al. Positive end-expiratory pressure: how to set it at the individual level. Ann Transl Med. 2017;5(14):288.

33. O'Driscoll B, et al. British Thoracic Society Guideline for oxygen use in adults in healthcare and emergency settings. BMJ Open Respir Res. 2017;4(1):e000170

34. Poor H. Basics of mechanical ventilation. Berlin: Springer; 2018.

35. NetworkTARDS. Ventilation with lower tidal volumes as compared with traditional tidal volumes for acute lung injury and the acute respiratory distress syndrome. N Engl J Med. 2000;342(18):1301-8.

36. Chiew YS, et al. Model-based PEEP optimisation in mechanical ventilation. Biomed Eng Online. 2011;10(1):111.

37. Morton SE, et al. Predictive virtual patient modelling of mechanical ventilation: impact of recruitment function. Ann Biomed Eng. 2019;47(7):1626-41.

38. Kim KT, et al. Quantifying neonatal pulmonary mechanics in mechanical ventilation. Biomed Signal Process Control. 2019;52:206-17.

39. Bates JH. Lung mechanics: an inverse modeling approach. Cambridge: Cambridge University Press; 2009.

40. Chase JG, et al. Next-generation, personalised, model-based critical care medicine: a state-of-the-art review of in silico virtual patient models, methods, and cohorts, and how to validation them. Biomed Eng Online. 2018;17:24.

41. Chase JG, et al. Digital twins in critical care: what, when, how, where, why? IFAC-PapersOnLine. 2021;54(15):310-5.

42. Arunachalam GR, et al. Virtual mechanical ventilation protocol—a model-based method to determine MV settings. IFAC-PapersOnLine. 2020;53(2):16119-24.

43. Lee JWW, et al. Protocol conception for safe selection of mechanical ventilation settings for respiratory failure Patients. Comput Methods Progr Biomed. 2022;214:106577.

44. Arnal J-M, et al. Parameters for simulation of adult subjects during mechanical ventilation. Respir Care. 2018;63(2):158-68.

\section{Publisher's Note}

Springer Nature remains neutral with regard to jurisdictional claims in published maps and institutional affiliations.

Ready to submit your research? Choose BMC and benefit from:

- fast, convenient online submission

- thorough peer review by experienced researchers in your field

- rapid publication on acceptance

- support for research data, including large and complex data types

- gold Open Access which fosters wider collaboration and increased citations

- maximum visibility for your research: over $100 \mathrm{M}$ website views per year

At BMC, research is always in progress.

Learn more biomedcentral.com/submissions 